\title{
Editorial: Data-Driven MHD - Novel Applications to the Solar Atmosphere
}

\begin{abstract}
A. K. Srivastava ${ }^{1 *}$, R. Erdélyi ${ }^{2,3,4}$, S. Poedts ${ }^{5,6}$, P. F. Chen ${ }^{7,8}$ and Y. Yan ${ }^{9}$
${ }^{1}$ Department of Physics, Indian Institute of Technology (BHU), Varanasi, India, ${ }^{2}$ Solar Physics and Space Plasma Research Centre, University of Sheffield, Sheffield, United Kingdom, ${ }^{3}$ Department of Astronomy, Eötvös Loránd University, Budapest, Hungary, ${ }^{4}$ Gyula Bay Zoltán Solar Observatory (GSO), Hungarian Solar Physics Foundation (HSPF), Gyula, Hungary, ${ }^{5}$ Centre for Mathematical Plasma Astrophysics, Department of Mathematics, KU Leuven, Leuven, Belgium, ${ }^{6}$ Institute of Physics, University of Maria Curie-Skłodowska, Lublin, Poland, ${ }^{7}$ School of Astronomy and Space Science, Nanjing University, Nanjing, China, ${ }^{8}$ Key Lab of Modern Astronomy and Astrophysics (Nanjing University), Ministry of Education, Nanjing, China, ${ }^{9}$ National Astronomical Observatory, Chinese Academy of Sciences, Beijing, China
\end{abstract}

Keywords: magnetohydrodynamics, corona, MHD waves, magnetic field, flares, Sun

Editorial on the Research Topic

Data-driven MHD: Novel Applications to the Solar Atmosphere

\section{INTRODUCTION}

The joint action of the complex magnetic field and ionised hot plasma leads to a range of dynamical processes, including magnetohydrodynamic (MHD) waves in the Sun's atmosphere at disparate spatial, temporal and spectral scales (Andries et al., 2009; Mathioudakis et al., 2013; Li et al., 2020; Van Doorsselaere et al., 2020; Srivastava et al., 2021; Wang et al., 2021). Different observatories have been employed since past several decades, both in space and at the ground, which put up a notably revamped knowledge about the physical processes transporting energy and mass in the Sun's magnetised atmosphere at diverse spatio-temporal scales (De Pontieu et al., 2004; Jess et al., 2009; Srivastava et al., 2017; Grant et al., 2018; Srivastava et al., 2018; Liu et al., 2019; Li et al., 2020; Van Doorsselaere et al., 2020). Remarkably, at the large spatial-scales, the key progress in understanding the origin of impulsive transient/eruptive phenomena such as energetic flares and bulky coronal mass ejections [CMEs, (Chen, 2011),], the related plasma processes detected at multi-wavelength radiative emissions varying from Gamma rays, X-rays to radio wave frequencies, as well as their space weather reverberations have illustrated a cardinal significance in the context of magnetohydrodynamics (MHD) modelling in recent era (Guo et al., 2019; Korsós et al.; Millas et al.; Samara et al., 2021). Besides the evolution of current age telescopes and their back-end cutting edge instruments for observing the dynamical plasma processes, noteworthy progress has been made in the theory of MHD waves, magnetic instabilities, global and local configurations of the magnetic fields and their energy build-up/release processes in the solar atmosphere. The novel science is emerged in understanding the wave processes, spontaneous/forced reconnection, and eruptive phenomena in the context of MHD. The leap forward advancements in the observations, coupled with theory, have emerged in form of front-line scientific progress in the field of solar astrophysics. These progresses have put down the basic preliminaries for current (e.g., 4m-DKIST (Rimmele et al., 2020; Rast et al., 2021); Parker Solar Probe (Bale et al., 2016); Solar Orbiter (Müller et al., 2020; GarcíaMarirrodriga et al., 2021)) and forthcoming high resolution new generation observatories (e. g., 4m-EST (Jurčák et al., 2019); 2.5m-WeHoT (Fang et al., 2019); 2m-NLST (Hasan et al., 2010); AdityaL1 (Raghavendra Prasad et al., 2017; Tripathi et al., 2017), CHASE (Li et al., 2019), etc.) to explore 
extensively the magnetic coupling of different layers of the Sun's atmosphere, their localised energy and mass transport phenomena, physical processes supporting a variety of eruptive events and space weather. The large cohort of observational data, once assimilated, will pave the way to realistic and stringent MHD modelling, whose novel scientific results may further match with the observations to solve numerous outstanding problems in solar and heliospheric physics.

The present special research topic has provided an opportunity to the solar physicists and space scientists to publish timely review articles and original novel scientific results on the exclusive theme of "Data-driven MHD: Novel Applications to the Solar Atmosphere". The guest editors solicited the scientific articles that put forward the way to the front-line observational data and associated data-driven MHD modelling collectively to answer the fundamental but earnest topics of the solar atmosphere. The objective of this topical issue is to put forward a novel insights into the solar and heliospheric physics community, particularly when the epoch of fine spatio-temporal resolution observations is on our horizon (Erdélyi et al., 2019; Erdélyi et al.,; Matthews et al., 2019; McCrea et al., 2019; Rast et al., 2021). Key scientific themes include, but are not limited to:

- The potential role of MHD waves in the hot and magnetized solar atmosphere (Pascoe et al.; Shukhobodskaia et al.);

- The dynamical plasma processes in the solar active regions, and their modelling in the framework of MHD (Jiang et al.);

- Prediction of the space weather candidates (e.g., solar flares and CMEs) (Korsós et al., 2021);

- Study of the MHD oscillations and solar magnetoseismology (SMS) utilizing cutting-edge photospheric, chromospheric, transition region and coronal observations from both space and ground (Pascoe et al.; Shukhobodskaia et al.);

- Modelling the large-scale solar eruptive and transient phenomena (e.g., flares, CMEs, solar wind) and their space weather and heliospheric consequences (Kumar et al.; Millas et al.).

The present special issue and collection of the articles bring a novel set of new scientific results, which are summarised in the next subsection.

\section{NOVEL SCIENTIFIC OUTCOMES OF THE TOPICAL ISSUE}

1) The complex plasma dynamics and energetics of the solar atmosphere are governed by the combined interplay of the plasma and magnetic fields. The current trends of the understanding of Sun's magnetic field origin and its data driven modelling are at the fore-front of the solar research, and there are extensive attempts made in recent days on this very important scientific theme (Bobra et al., 2008; Cheung and DeRosa, 2012; Kliem et al., 2013; Dalmasse et al., 2019; Zhou et al., 2020; Yardley et al., 2021). The paper titled “ $M H D$ Modelling of Solar Coronal Magnetic Evolution Driven by
Photospheric Flow" by Jiang et al. depicted a cutting edge perspective of the data-driven MHD simulations of magnetic fields generated in the solar active region (AR). To the extent of our knowledge, this is the first result to develop the observational data enabled full MHD model that utilises directly the flow field at the solar photosphere measured with the DAVE4VM technique. They establish an MHD equilibrium gleaned on one vector magnetogram by utilising an MHD-relaxation model with an adeptly less kinetic viscosity. They further apply the reported MHD equilibrium as an initial condition for ensuing a datadriven evolution. Thereafter, they derive the plasma flows at the solar photosphere from the time series of the observed magnetograms derived from DAVE4VM method. In the present study, the surface plasma flows are ultimately employed as an input in the time sequence to the bottom boundary of the reported MHD model. It concordantly upgrade the magnetic field at each time step by directly solving the magnetic induction equation at the bottom boundary of the numerical simulation box. They apply this MHD model to understand the generation of the solar magnetic field in AR 12158 whose sources are observed by SDO/HMI vector magnetograms in their paper. Their numerical model brings out a quasi-static stress of the magnetic line of forces primarily via the rotational flows of the leading sunspot of solar active region. This process creates the core magnetic field lines to constitute a coherent S-shape resemblance with the sigmoidal structure as often observationally detected in the solar atmosphere. The total magnetic energy acquired in the numerical model seen equalising closely to the stored magnetic energy as estimated straight away from the original vector magnetogram using the estimated optical flow fields by DAVE4VM. Such a new data-driven magnetic field model possesses potential scientific implications in the field of solar physics to study that how the Sun's magnetic fields, as driven by the slow plasma flow fields at the photosphere, get into an unstable state and run into the gigantic eruptions.

2) Kink mode oscillations are ubiquitously evident in a variety of magnetic structures in the solar atmosphere (Andries et al., 2009). In the paper titled "Oscillation and Evolution of Coronal Loops in a Dynamical Solar Corona”, Pascoe et al. (Pascoe et al.) perform the numerical simulations in order to understand the oscillation and evolution of solar coronal loops in the dynamical solar atmosphere. They have investigated the observational evidences of the magnetoacoustic kink oscillations and the KelvinHelmholtz $(\mathrm{KH})$ instability both using high-resolution seismological and spatial data analysis methods. The exclusive finding elucidates that low amplitude kink oscillations may play a role in giving rise the significant changes in the loop profile. This may influence the measurements of the transverse loop inhomogeneity based on the seismological and forward modelling methods. The influence on forward modelling estimates in the present work may also result for the previous observational signatures advocating loops having a wider 
inhomogeneous layer. Such diagnostics are very crucial to understand the diagnostics and in understanding the dissipative processes of such waves in coronal loops.

3) As stated in (Mathioudakis et al., 2013), the kink oscillations of the solar magnetic loops have been extensively explored, both in the observational data and in the theoretical models, since the last couple of decades. Recently, this is demonstrated that most of the observationally detected driven kink oscillations of the coronal loops are subjected to the damping with either exponential or Gaussian profiles. A variety of physical mechanisms/processes are now well studied to understand the damping phenomenon of the kink oscillations (Shukhobodskaia et al.). However, some of the driven kink oscillations are noted to be evolved in such a way that they may not be modelled by using a Gaussian or exponential damping profiles. This is due to the fact that the amplification of kink oscillations are even observed at several instances in the coronal loops. A variety of recent scientific results in this context have delineated that including the joint effects of coronal loop expansion, cooling, and resonant absorption may result in the significant departure from the Gaussian and exponential damping profiles of the fast magnetoacoustic kink oscillations. It significantly describes the increase in oscillation amplitude w.r.t. time in many observed cases. In the paper titled "Significance of Cooling Effect on Comprehension of Kink Oscillations of Coronal Loops" (Shukhobodskaia et al.), authors examined ten driven kink oscillations in the coronal loops to further probe on the ability of expansion and cooling to expound the complicated damping profiles of these oscillations. Their results do not reckon on fitting a periodicity to these transverse oscillations by considering the complexities in both the temporal (i.e., change in the period) and spatial (i.e., change in the amplitude) variations. This can be considered for in a sophisticated and easy course of action. Additionally, this approach may also permit authors to theorise some important diagnostic information (e.g., the density ratio at the loop footpoints where kink oscillations were evolved) from the oscillation profile alone. This diagnostics does not require any comprehensive measurements of the loop as well as complex numerical modelling. The derived scientific results suggest the correlations between the density ratio at the loop footpoints and the amplitudes and periods of the kink oscillations. Ultimately, the authors compare their obtained results to the previous models, specifically the purely Gaussian and exponential damping profiles of the kink oscillations, through the estimations of $\Xi^{2}$ values. Therefore, they find that the inclusion of cooling can produce better fits to the damped kink oscillations in some cases. The present scientific findings suggest that the temperature evolution must be incorporated in the kink-mode oscillation models to best understand these oscillations which are not purely Gaussian or exponential in nature.

4) It is extensively studied in the literature that the most dynamic solar active-regions (ARs) are well-known to give rise the gigantic flares rapidly, where this frequency varies with the solar cycle. The prediction of these flares is a difficult task, and a wide range of methodologies is applied to proceed. Machine learning approaches seem to have strong potentials (Bobra and Couvidat, 2015; Kusano et al., 2020). In this topical issue the paper titled "Testing and Validating Two Morphological Flare Predictors by Logistic Regression Machine Learning" (Korsós et al.) addresses a stringent method that is developed to investigate and assess numerical measures of the mixed states of ARs with opposite magnetic field polarities. By evaluating two morphological parameters, i.e., the separation parameter, as well as the sum of the horizontal magnetic field gradient, the present study delivers engrossing evidences for the hypothesised relation between the level of mixed states of the studied active region (AR) and the level of the solar eruption probability within it. The efficiency of these two parameters as flare predictors, or flare-pre-cursors, is tested on a archetypal sample of randomly selected ARs, based on the SOHO/MDI-Debrecen Data (SDD) and the SDO/HMI - Debrecen Data (HMIDD) sunspot catalogues. The selected data satisfy well-defined selection criteria. Over 1,000 ARs were analysed to confirm the combined forecasting abilities of these two morphological parameters. The logistic regression machine learning method was employed to carry out the study. It is shown that the two pre-cursors with their threshold values act as excellent interrelated predictors. The conditional forecasting probability of the investigated precursors is found to be at least $70 \%$ a day ahead of flare onset. This is certainly an improvement when compared to the currently available similar studies.

5) The space weather refers to the physical conditions in the interplanetary medium and in the solar terrestrial environment. It is basically generated by the solar activity, inclusive of the variable ambient solar wind, energetic flares, and huge and massive coronal mass ejections (CMEs) (Guo et al., 2019; Korsós et al., 2020; Millas et al.; Samara et al., 2021). Mitigation of the adverse space weather effects with economic and social benefits needs an improved knowledge of the Sun-Earth relation. Data and models are the basis for effective space weather forecasting. The paper entitled "Domain of Influence Analysis: Implications for Data Assimilation in Space Weather Forecasting" (Millas et al.), contains a description of the famous and frequently utilised space weather forecast models as well as the most suitable locations for space weather data gathering. The authors used three models that simulate different stages of the connection of the Sun to the Earth and applied Representer Analysis (RA) on them. They also performed a Domain of Influence (DOI) analysis to the same models. The models considered are OpenGGCM and Tsyganenko, which focus on the interplay of the supersonic solar wind with the planetary magnetosphere, and PLUTO which is applied to numerically model the propagation of the coronal mass ejections (CMEs) in the interplanetary space. Their model based analysis is favourable for the studies of space weather dynamics for the multitude of reasons. On the first instance, they get quantitative understanding of the most utilitarian locations of the observation points, e.g., solar wind monitors. For the illustration, they describe that the absolute values of 
the Domain of Influence (DOI) are exceptionally lower in the magnetospheric plasma sheet at the Earth's outer atmosphere. Given the fact that the understanding of this specific subsystem is pivotal for the space weather, an effective and augmented monitoring of the region will be highly advantageous. On the other hand, they are accomplished to better characterise the numerical models. Even if the present analysis aims on spatial correlations compared to the temporal correlations, they infer that the time-independent numerical models are less useful for the Data Assimilation pursuit compared to the time-dependent numerical models. Finally, they get hold of the first exclusive stride toward enterprising objectives of pin pointing the most pertinent heliospheric parameters for modelling the CME propagation in the heliosphere, their arrival time, and exclusively their geoeffectiveness at the Earth's magnetosphere. The present work clearly improves the data acquisition and modelling capabilities of the interplanetary responses of the spaceweather candidates.

6) CMEs and sporadic solar jets set out as perturbations to the ambient supersonic solar wind, and their joint effects exhibit a vital inference in the space weather associated dynamical plasma processes. Consequently, a vigorous foundation for precise forecast of the background solar wind properties is a most essential pathway towards the progress of any space weather prediction. In the paper titled "A Comparison Study of Extrapolation Models and Empirical Relations in Forecasting Solar Wind" (Kumar et al.), the authors studied on the application and comparison of different numerical models which are crucial to the prediction of a steady state high-speed solar wind. Notably, the authors carry out the case studies on Carrington rotations 2053, 2082, and 2104. Thereafter, they have evaluated the performance of magnetic field extrapolation models in concurrence with the velocity empirical formulations. This further helps in predicting the physical properties of the solar wind at the Lagrangian point "L1". The two completely distinct models to extrapolate the solar wind from the Sun's corona to the innerheliospheric space are described by the authors. The first model is related with the kinematics based Heliospheric Upwind eXtrapolation (HUX) model. The second model is a physics based model. With the PLUTO open-source code, the physics-based model solves a set of hydrodynamic equations in the conservative form, and the numerical results can also estimate and forecast the thermal parameters of the solar wind. The predictions of the solar wind parameters by different models are quantitatively assessed by comparing with statistical observations. Moreover, they extend such a developed modelling framework to forecast the polarities of interplanetary magnetic fields near the Earth. Their model performs pretty well, for example, the comparison between their predictions and the observations leads to a remarkable correlation coefficient up to $\sim 0.73-0.81$, with a root mean square error of $\sim 75-90 \mathrm{~km} \mathrm{~s}^{-1}$. In addition, they compare the physics-based model and the hourly time-scale OMNI solar wind data. It is found that the standard deviation is similar to each other and the solar wind proton temperatures match very well between predictions and in-situ measurements.

\section{CONCLUSION}

The present topical collection brings some state-of-art scientific articles on the study of physical understanding of the MHD wave modes and their behaviour in the non-adiabatic solar atmosphere; their numerical simulations in magnetic loops. Moreover, the stringent active region modelling and the flare prediction models provide novel scientific understanding on the dynamics of eruptive phenomena and their predictions in the framework of space weather research. A comparative study of the extrapolation models and empirical relations in predicting the solar wind also put forward a comprehensive study on magnetic extrapolation models and their role in understanding and forecasting the physical properties of the solar wind over the Carrington rotations. The role of the MHD modelling and data assimilation in space weather forecasting is also emphasised in this collection. Given the fact that new as well as forthcoming observatories from ground and space in the modern era of solar and heliospheric physics are focusing on understanding energy and mass transport processes at diverse spatio-temporal scales as well as space weather studies, the present topical issue serves significantly with some selective front-line research papers and their thematic contents in demonstrating the cutting-edge progress in these research fields.

\section{AUTHOR CONTRIBUTIONS}

All authors listed have made a substantial, direct, and intellectual contribution to the work and approved it for publication.

\section{FUNDING}

RE acknowledge to the Science and Technology Facilities Council (STFC, grant number ST/M000826/1) United Kingdom and the Royal Society for enabling this research. RE also acknowledges the support received by the CAS Presidents International Fellowship Initiative Grant No. 2019VMA052. PC is supported by NSFC (11961131002). AS and RE acknowledge the support from UKIERI (Indo-UK) Project Grant (2018-2021; 184-1/2018(IC)).

\section{ACKNOWLEDGMENTS}

RE also acknowledges the warm hospitality received at USTC, Hefei, where part of his contribution was made. 


\section{REFERENCES}

Andries, J., van Doorsselaere, T., Roberts, B., Verth, G., Verwichte, E., and Erdélyi, R. (2009). Coronal Seismology by Means of Kink Oscillation Overtones. Space Sci. Rev. 149, 3-29. doi:10.1007/s11214-009-9561-2

Bale, S. D., Goetz, K., Harvey, P. R., Turin, P., Bonnell, J. W., Dudok de Wit, T., et al. (2016). The FIELDS Instrument Suite for Solar Probe Plus. Space Sci. Rev. 204, 49-82. doi:10.1007/s11214-016-0244-5

Bobra, M. G., and Couvidat, S. (2015). Solar Flare Prediction Usingsdo/hmi Vector Magnetic Field Data with a Machine-Learning Algorithm. ApJ 798, 135. doi:10.1088/0004-637X/798/2/135

Bobra, M. G., van Ballegooijen, A. A., and DeLuca, E. E. (2008). Modeling Nonpotential Magnetic Fields in Solar Active Regions. ApJ 672, 1209-1220. doi:10.1086/523927

Chen, P. F. (2011). Coronal Mass Ejections: Models and Their Observational Basis. Living Rev. Solar Phys. 8, 1. doi:10.12942/lrsp-2011-1

Cheung, M. C. M., and DeRosa, M. L. (2012). A Method for Data-Driven Simulations of Evolving Solar Active Regions. ApJ 757, 147. doi:10.1088/0004-637X/757/2/147

Dalmasse, K., Savcheva, A., Gibson, S. E., Fan, Y., Nychka, D. W., Flyer, N., et al. (2019). Data-optimized Coronal Field Model. I. Proof of Concept. ApJ 877, 111. doi: $10.3847 / 1538-4357 / a b 1907$

De Pontieu, B., Erdélyi, R., and James, S. P. (2004). Solar Chromospheric Spicules from the Leakage of Photospheric Oscillations and Flows. Nature 430, 536-539. doi:10.1038/nature02749

Erdélyi, R., Amari, T., Belucz, B., Berrilli, F., Bogachev, S., Bolsée, D., et al. (2019). HiRISE - High Resolution Imaging and Spectroscopy Explorer - Ultrahigh Resolution, Interferometric and External Occulting Coronagraphic Science: Great Leap in Solar Physics. ESA Voyage. 2050 White Paper.

Erdélyi, R., Damé, L., Fludra, A., Mathioudakis, M., Amari, T., Belucz, B., et al. . HiRISE - High-Resolution Imaging and Spectroscopy Explorer - Ultrahigh Resolution, Interferometric and External Occulting Coronagraphic Science. J. Exp. Astron. (under review).

Fang, C., Gu, B., Yuan, X., Ding, M., Chen, P., Dai, Z., et al. (2019). 2.5 M WideField and High-Resolution Telescope. Sci. Sin.-Phys. Mech. Astron. 49, 059603. doi:10.1360/SSPMA2018-00313

García Marirrodriga, C., Pacros, A., Strandmoe, S., Arcioni, M., Arts, A., Ashcroft, C., et al. (2021). Solar Orbiter: Mission and Spacecraft Design. AઐA 646, A121. doi:10.1051/0004-6361/202038519

Grant, S. D. T., Jess, D. B., Zaqarashvili, T. V., Beck, C., Socas-Navarro, H., Aschwanden, M. J., et al. (2018). Alfvén Wave Dissipation in the Solar Chromosphere. Nat. Phys. 14, 480-483. doi:10.1038/s41567-018-0058-3

Guo, Y., Xia, C., Keppens, R., Ding, M. D., and Chen, P. F. (2019). Solar Magnetic Flux Rope Eruption Simulated by a Data-Driven Magnetohydrodynamic Model. ApJ 870, L21. doi:10.3847/2041-8213/aafabf

Hasan, S. S., Soltau, D., Kärcher, H., Süß, M., and Berkefeld, T. (2010). NLST: India's National Large Solar Telescope. Astron. Nachr. 331, 628-635. doi:10.1002/ asna.201011389

Jess, D. B., Mathioudakis, M., Erdélyi, R., Crockett, P. J., Keenan, F. P., and Christian, D. J. (2009). Alfven Waves in the Lower Solar Atmosphere. Science 323, 1582-1585. doi:10.1126/science.1168680

Jurčák, J., Collados, M., Leenaarts, J., van Noort, M., and Schlichenmaier, R. (2019). Recent Advancements in the EST Project. Adv. Space Res. 63, 1389-1395. doi:10.1016/j.asr.2018.06.034

Kliem, B., Su, Y. N., van Ballegooijen, A. A., and DeLuca, E. E. (2013). Magnetohydrodynamic Modeling of the Solar Eruption on 2010 April 8. ApJ 779, 129. doi:10.1088/0004-637x/779/2/129

Korsós, M. B., Georgoulis, M. K., Gyenge, N., Bisoi, S. K., Yu, S., Poedts, S., et al. (2020). Solar Flare Prediction Using Magnetic Field Diagnostics above the Photosphere. ApJ 896, 119. doi:10.3847/1538-4357/ab8fa2

Kusano, K., Iju, T., Bamba, Y., and Inoue, S. (2020). A Physics-Based Method that Can Predict Imminent Large Solar Flares. Science 369, 587-591. doi:10.1126/science.aaz2511

Li, B., Antolin, P., Guo, M.-Z., Kuznetsov, A. A., Pascoe, D. J., Van Doorsselaere, T., et al. (2020). Magnetohydrodynamic Fast Sausage Waves in the Solar Corona. Space Sci. Rev. 216, 136. doi:10.1007/s11214-020-00761-z

Li, C., Fang, C., Li, Z., Ding, M.-D., Chen, P.-F., Chen, Z., et al. (2019). Chinese Ha Solar Explorer (CHASE) - a Complementary Space mission to the ASO-S. Res. Astron. Astrophys. 19, 165. doi:10.1088/1674-4527/19/11/165
Liu, J., Nelson, C. J., Snow, B., Wang, Y., and Erdélyi, R. (2019). Evidence of Ubiquitous Alfvén Pulses Transporting Energy from the Photosphere to the Upper Chromosphere. Nat. Commun. 10, 3504. doi:10.1038/s41467-019-11495-0

Mathioudakis, M., Jess, D. B., and Erdélyi, R. (2013). Alfvén Waves in the Solar Atmosphere. Space Sci. Rev. 175, 1-27. doi:10.1007/s11214-012-9944-7

Matthews, S., Baker, D., Bloomfield, D. S., Browning, P. K., Calcines, A., Del Zanna, G., et al. (2019). Solar Particle Acceleration, Radiation \& Kinetics (SPARK). ESA Voyage 2050 White Paper.

McCrea, I., Davies, J., Dunlop, M., Erdélyi, R., Forsyth, C., Harra, L., et al. (2019). "The Grand European Heliospheric Observatory" - an Integrated ESA Approach to Challenges in Solar and Solar-Terrestrial Physics. ESA Voyage 2050 White Paper.

Müller, D., St. Cyr, O. C., Zouganelis, I., Gilbert, H. R., Marsden, R., NievesChinchilla, T., et al. (2020). The Solar Orbiter mission. AઐAA\&A 642, A1. doi:10.1051/0004-6361/202038467

Raghavendra Prasad, B., Banerjee, D., Singh, J., Nagabhushana, S., Kumar, A., Kamath, P. U., et al. (2017). Visible Emission Line Coronagraph on Aditya-L1. Curr. Sci. 113, 613. doi:10.18520/cs/v113/i04/613-615

Rast, M. P., Bello González, N., Bello González, N., Bellot Rubio, L., Cao, W., Cauzzi, G., et al. (2021). Critical Science Plan for the Daniel K. Inouye Solar Telescope (DKIST). Sol. Phys. 296, 70. doi:10.1007/s11207-021-01789-2

Rimmele, T. R., Warner, M., Keil, S. L., Goode, P. R., Knölker, M., Kuhn, J. R., et al. (2020). The Daniel K. Inouye Solar Telescope - Observatory Overview. S Solar Phys. 295, 172. doi:10.1007/s11207-020-01736-7

Samara, E., Pinto, R. F., Magdalenić, J., Wijsen, N., Jerčić, V., Scolini, C., et al. (2021). Implementing the MULTI-VP Coronal Model in EUHFORIA: Test Case Results and Comparisons with the WSA Coronal Model. A\&A 648, A35. doi:10.1051/0004-6361/202039325

Srivastava, A. K., Ballester, J. L., Cally, P. S., Carlsson, M., Goossens, M., Jess, D. B., et al. (2021). Chromospheric Heating by Magnetohydrodynamic Waves and Instabilities. J. Geophys. Res. Space Phys. 126, e029097. doi:10.1029/2020JA029097

Srivastava, A. K., Murawski, K., Kuźma, B., Wójcik, D. P., Zaqarashvili, T. V., Stangalini, M., et al. (2018). Confined Pseudo-shocks as an Energy Source for the Active Solar corona. Nat. Astron. 2, 951-956. doi:10.1038/s41550-018-0590-1

Srivastava, A. K., Shetye, J., Murawski, K., Doyle, J. G., Stangalini, M., Scullion, E., et al. (2017). High-frequency Torsional Alfvén Waves as an Energy Source for Coronal Heating. Sci. Rep. 7, 43147. doi:10.1038/srep43147

Tripathi, D., Ramaprakash, A. N., Khan, A., Ghosh, A., Chatterjee, S., Banerjee, D., et al. (2017). The Solar Ultraviolet Imaging Telescope On-Board Aditya-L1. Curr. Sci. 113, 616. doi:10.18520/cs/v113/i04/616-619

Van Doorsselaere, T., Srivastava, A. K., Antolin, P., Magyar, N., Vasheghani Farahani, S., Tian, H., et al. (2020). Coronal Heating by MHD Waves. Space Sci. Rev. 216, 140. doi:10.1007/s11214-020-00770-y

Wang, T., Ofman, L., Yuan, D., Reale, F., Kolotkov, D. Y., and Srivastava, A. K. (2021). Slow-Mode Magnetoacoustic Waves in Coronal Loops. Space Sci. Rev. 217, 34. doi:10.1007/s11214-021-00811-0

Yardley, S. L., Mackay, D. H., and Green, L. M. (2021). Simulating the Coronal Evolution of Bipolar Active Regions to Investigate the Formation of Flux Ropes. Sol. Phys. 296, 10. doi:10.1007/s11207-020-01749-2

Zhou, Y. H., Chen, P. F., Hong, J., and Fang, C. (2020). Simulations of Solar Filament fine Structures and Their Counterstreaming Flows. Nat. Astron. 4, 994-1000. doi:10.1038/s41550-020-1094-3

Conflict of Interest: The authors declare that the research was conducted in the absence of any commercial or financial relationships that could be construed as a potential conflict of interest.

Publisher's Note: All claims expressed in this article are solely those of the authors and do not necessarily represent those of their affiliated organizations, or those of the publisher, the editors and the reviewers. Any product that may be evaluated in this article, or claim that may be made by its manufacturer, is not guaranteed or endorsed by the publisher.

Copyright $\odot 2021$ Srivastava, Erdélyi, Poedts, Chen and Yan. This is an open-access article distributed under the terms of the Creative Commons Attribution License (CC BY). The use, distribution or reproduction in other forums is permitted, provided the original author(s) and the copyright owner(s) are credited and that the original publication in this journal is cited, in accordance with accepted academic practice. No use, distribution or reproduction is permitted which does not comply with these terms. 